\title{
¿Progresa Adecuadamente la Justicia en Andalucía?
}

\author{
Is Spanish Justice Satisfactorily Progressing in Andalusia? \\ María José Vázquez-Cueto \\ pepi@us.es O https://orcid.org/0000-0003-1695-7723 \\ Facultad de Ciencias Económicas y Empresariales. Universidad de Sevilla. \\ Avenida Ramón y Cajal 1. Sevilla 41018 \\ Francisco Gutiérrez-López \\ f.gutierrez@poderjudicial.es; francisco.gutierrez.ius@juntadeandalucia.com @ https://orcid.org/0000-0002-2948-4286 \\ Sección Cuarta de la Audiencia Provincial de Sevilla. \\ Prado de San Sebastián s/n. 41071 Sevilla.
}

\section{INFO ARTÍCULO}

Recibido: 27-09-2019

Revisado: 30-04-2020

Aceptado: 22-05-2020

\section{PALABRAS CLAVE}

Administración de Justicia

Gráficos radiales

Andalucía

España

Sector público regional

\section{KEYWORDS}

Administration of Justice

Radar charts

Andalusia

Spain

Regional public sector

\section{RESUMEN}

La Administración del Estado, consciente de la importancia del buen funcionamiento del Sistema Judicial de un país, le provee de los recursos necesarios. Sin embargo, su reparto entre las distintas Comunidades Autónomas (CCAA) provoca desigualdades entre las mismas. Centrándose en la CA de Andalucía, este trabajo tiene como objetivo comparar la evolución del funcionamiento de su sistema judicial con el del resto de España. Dada las peculiaridades de cada Jurisdicción, se realiza el análisis a nivel global y por Jurisdicción. Mediante la técnica de los gráficos radiales, se observan las mejoras producidas en los periodos 2009-2012, 2012-2015 y 2016-2018, situando a Andalucía en el contexto nacional. Los resultados muestran que, a nivel global y para el periodo 2009-2012, Andalucía sigue el mismo camino de mejora que el resto de España, y sin embargo la situación andaluza se deteriora para el periodo 2012-2015, siendo la Jurisdicción civil la más afectada. Con esta técnica, que no ha sido hasta el momento aplicada a la Justicia, se abre un campo nuevo en la investigación de un sector público cuyo funcionamiento condiciona al de otros y tiene implicaciones directas en la economía de un país.

\begin{abstract}
The state's administration, concerned about the importance of having an efficient Judicial System attempt to provide it with the required resources. Nevertheless, due to the state division in autonomies remarkable inequalities are produced between them. Along this essay, principally focused in Andalusian autonomous community, a comparison of the development of its judicial system efficiency will be analysed along with the rest of Spain. Given the specificities of each jurisdiction, the analysis will be done at a global level and later, specified in each jurisdiction. Via the radar chart's technique improvements will be analysed throughout the following periods, 2009-2012, 2012-2015 y 2016-2018, placing Andalusia in the national context. The results show that, globally in the 2009-2012 period, Andalusia follows the improvement path as well as the rest of Spain, notwithstanding the Andalusian situation decays in the following period, 2012-2015, being the civil jurisdiction the most affected one. With an unprecedented use of this technique in justice, a new investigation field is discovered to analyse the public sector, whose proper function conditionate other ones and has direct economic implications for the country.
\end{abstract}




\section{INTRODUCCIÓN}

El funcionamiento de la Administración de Justicia en España está intensamente cuestionado en la actualidad. Investigaciones penales interminables, juicios señalados a años vista y un sinfín de disfunciones cuestionan su eficacia ante los profesionales de la justicia y ante la ciudadanía.

La desconfianza social en el funcionamiento del sistema judicial español es elevada y ha empeorado en los últimos años. Basta consultar los datos que ofrece el CIS sobre la percepción que tiene la ciudadanía para constatarlo. Así, a la pregunta “¿En qué medida: muy, bastante, poco o nada; diría Ud. que cada uno de los siguientes servicios públicos funcionan satisfactoriamente? Particularmente, para la Administración de Justicia en 2007 el 61,8\% contestaban que poco o nada, porcentaje que subía hasta el 73,1\% en 2017.

Las disfunciones del sistema judicial no solo ponen en entredicho su funcionamiento sino también su sistema económico (Pastor y Rosales, 2005) y social por la pérdida de seguridad jurídica que provocan y las consecuencias que acarrean, como ya señaló Santos Pastor Prieto en 2015.

Esta situación no es desconocida por el poder político, pues ya en mayo del 2001 el Partido Popular (PP), el Partido Socialista Obrero Español (PSOE) y el gobierno de la nación firmaron el Pacto para la Reforma de la Justicia, reconociéndose así, como una cuestión de Estado, la necesidad de modernizar la Administración de Justicia en España para que desarrollara eficazmente sus funciones constitucionales. Sin embargo, dieciocho años después, pese a las medidas que se propusieron en dicho Pacto y al esfuerzo presupuestario desplegado estos años, no parece que el objetivo se haya alcanzado o, al menos, así lo perciben los ciudadanos. Ahora bien, ¿es esta una percepción mediatizada o está basada en datos objetivos? El presente trabajo trata de dar respuesta a esta cuestión.

Dada la peculiaridad del Sistema Judicial Español, que tiene transferidas partes de sus competencias a las Comunidades Autónomas (CCAA), el funcionamiento difiere en cada una de ellas. El análisis se centra en la Comunidad Autónoma de Andalucía para la que se analiza la mejora de su sistema judicial comparándola con la del resto de España. Se utiliza como periodo de referencia los años comprendidos entre 2009 y 2018. Con el objetivo no solo de analizar las potenciales mejoras de su sistema judicial sino también la evolución de las mismas, se descompone el periodo en tres subperiodos, presentando los resultados de las mejoras experimentadas entre los años 2009 a 2012, 2012 a 2015 y 2016 a 2018. El primero de ellos se corresponde con un periodo de crisis económica y financiera que afectó a todos los estamentos de la sociedad española y repercutió en las partidas presupuestarias asignadas a los sectores públicos, entre ellos a la Justicia, a esto se une una mayor tasa de criminalidad derivada de la situación económica de los ciudadanos y un mayor número de litigios procedentes de quiebras empresariales. 2012-2015 es un periodo en el que la economía empieza a remontar y los indicadores macroeconómicos, aunque no llegan a alcanzar los niveles anteriores a la crisis, sí que muestran una senda de optimismo, pudiendo considerarse, pues, como un periodo de relativa estabilidad. El último de los periodos considerados es de particular interés ya que en 2015 se modificó el cómputo de los asuntos ingresados, haciendo incomparables las series temporales de las tasas que se utilizan.

La determinación de las razones que han dado lugar a los resultados obtenidos se escapa del objetivo de este trabajo, que pone su énfasis en las desigualdades territoriales que se producen en el funcionamiento de la justicia tomando como referencia a la Comunidad Autónoma de Andalucía. Para ello, y tras esta introducción que brevemente acota el problema a tratar, se dedica el apartado dos a comentar el complejo sistema judicial español, como elemento clave en el funcionamiento de este. En el apartado tres se presenta la metodología que se utiliza en el estudio, y que se aplica a las variables definidas en el apartado cuatro. El apartado cinco presenta los resultados obtenidos para todas las Jurisdicciones, y su desglose por Jurisdicción. Se termina con la discusión y conclusiones en el apartado seis. 


\section{EL SISTEMA JUDICIAL ESPAÑOL Y SU DESCENTRALIZACIÓN EN CCAA}

Pese a que la Constitución Española estableció en su artículo 149, 1-5 que la Administración de Justicia era competencia exclusiva del Estado, el desarrollo legislativo posterior y la interpretación que realizó el Tribunal Constitucional en sus sentencias $n^{\circ} 56$ y 62/1990 del precepto lo han matizado.

Se distingue entre Administración de Justicia en sentido estricto, que es el Poder Judicial, que no se puede transferir, y, por otro lado, lo que denominó "la administración de la Administración de Justicia", encargada de proveer de medios materiales y personales a la primera, que sí se puede transferir.

Ello ha conformado una Administración de Justicia desconcentrada, en la que las competencias de la Administración Central están repartidas entre el Ministerio de Justicia (MJ), teniendo algunas competencias residuales sobre la Carrera Fiscal la Fiscalía General del Estado, y el Consejo General el Poder Judicial (CGPJ), y descentralizada, pues algunas competencias (funcionarios, oficina judicial, medios materiales y justicia gratuita) se pueden transferir a las Comunidades Autónomas (CCAA). Esto explicaría que en un mismo órgano judicial puedan coexistir hasta tres administraciones diferentes y, por ejemplo, que cohabiten en todo el territorio nacional hasta nueve sistemas operativos informáticos incompatibles entre ellos. Problema que se ve acentuado por la falta de un organismo centralizador o director de la organización judicial.

El funcionamiento de la administración de Justicia en España es, por tanto, un concepto que engloba el funcionamiento de la administración de justicia en sus CCAA. Además, en nuestro sistema judicial se distinguen cuatro jurisdicciones: penal, civil, contencioso- administrativa y social o laboral. La penal, encargada de investigar, juzgar y ejecutar las conductas que son calificadas expresamente como delitos, es la que consume más recursos; la civil, competente para enjuiciar y ejecutar las controversia entre particulares, consume, también, gran cantidad de recursos, especialmente en la ejecución; la jurisdicción contencioso-administrativa, enjuicia y ejecuta las controversias del ciudadano con la administración, tiene una incidencia menor; y la jurisdicción social o laboral, que es la competente para enjuiciar y ejecutar las controversias entre empresarios y trabajadores y, también, las relacionadas con la Seguridad Social. Según datos del CGPJ, para 2016, la jurisdicción penal registró el 60\% de los asuntos ingresados, el 30\% la jurisdicción civil, el 3\% la jurisdicción contenciosa-administrativa y el $6 \%$ la social.

Pese a la descentralización, la actividad jurisdiccional de los juzgados y tribunales está organizada por Tribunales Superiores de Justicia (TSJ), uno por CCAA, salvo en el caso de Andalucía que tiene integrada las Ciudades de Ceuta y Melilla (Tribunal Superior de Justicia de Andalucía, Ceuta y Melilla), y la información que actualmente ofrece el servicio de Estadística Judicial del CGPJ sobre la actividad judicial es muy completa, lo que permite analizar el funcionamiento de la Administración de Justicia en España y en sus CCAA separadamente. Y no solo a nivel global, sino también por Jurisdicciones.

\section{METODOLOGÍA}

En este trabajo se utiliza la técnica de los gráficos radiales. Es fundamentalmente una técnica de visualización, que ha sido utilizada en otros campos como, por ejemplo, en la metodología de evaluación medioambiental (Aldunce, Neri y Szlafsztein, 2008), en el análisis de la gestión empresarial (Moerke, 1997; Pérez Benedito y Ángel, 2011), en sectores productivos específicos (sector pesquero) y ampliamente como complementaria a otras técnicas contables (Pérez, 2016), e incluso en la evaluación de sectores públicos tales como el transporte (Pérez, 2013). Las primeras aplicaciones comparaban las mejoras que se producían en los mercados laborales europeos midiendo variables relacionadas con el empleo (Schütz, Speckesser y Schmid, 1998; Mosley y Mayer, 1999), y más recientemente Bazzani et al. en 2018 también han tratado el empleo con esta técnica. Sin embargo, en nuestro conocimiento, no ha sido hasta el momento aplicada a la Justicia. En este sentido, se abre un campo nuevo en la investigación de un sector público cuyo funcionamiento condiciona al de otros sectores y tiene implicaciones directas en la economía de un país.

Un gráfico radial es la representación de datos mediante ejes radiales. Cualquier software de tratamiento de datos, aunque no sea muy sofisticado, puede proporcionarlo, siempre y cuando se introduzcan los 
valores de manera apropiada. Si se caracteriza un objeto en función de varios atributos medibles, el gráfico radial ofrecerá su representación visual utilizando cada eje para indicar el valor de un atributo específico. Estos valores habrá previamente que estandarizarlos pues, en caso contrario, los ejes no serían comparables. Uniendo estos valores se obtiene un polígono irregular representativo del perfil del objeto en función de los atributos considerados. Esto permitirá comparar objetos entre sí, o bien la evolución de un mismo objeto a lo largo del tiempo o del espacio.

Fijada una región y elegidas las magnitudes que la caracterizan, se puede, por ejemplo, representar los valores de estas en dos periodos de tiempo y, de esta forma, visualizar su evolución. También pueden utilizarse para realizar análisis espaciales: calculados los valores de las magnitudes caracterizadoras de las regiones en un determinado momento, representarlos para varias regiones $\mathrm{y}$, visualmente, poder comparar unas con otras.

Obviamente cuanto mayor número de ejes considere el gráfico, mejor será la representación de la realidad de la situación, pero, al ser una de sus principales ventajas la visualización conjunta, a mayor número de ejes se irá perdiendo este poder. Hay que combinar, pues, cuidadosamente un suficientemente representativo número de ejes con una buena visualización. Como complemento a esta visualización, a través del polígono irregular formado por el objeto, puede elaborarse un indicador que lo represente: El área. Cuanto mayor sea el valor del área, mejor será la situación del objeto analizado.

Para este estudio concreto: la mejora del funcionamiento de la Administración de Justicia en la Comunidad Autónoma de Andalucía (Andalucía) situada en el contexto nacional, se consideran cuatro indicadores claves según el CGPJ. Estos son la tasa de litigiosidad, tasa de resolución, tasa de pendencia y tasa de congestión, definidas en la tabla 1.

Adaptando el trabajo de Mosley y Mayer (1999), a este caso concreto, se siguen los siguientes pasos. Definidas las variables indicativas del funcionamiento de la Justicia, se obtienen sus valores para Andalucía y para todo el territorio nacional excluyendo Andalucía (España) en los años 2009, 2012, 2015, 2016 y 2018. A continuación, se procede a definir las direcciones de mejora estandarizadas y se calculan para el análisis de las mejoras producidas en los periodos 2009 a 2012, 2012 a 2015 y 2016 a 2018. Para cada uno de estos periodos se representan las mejoras en un gráfico radial, en el que en cada eje recoge la mejora de una de las variables consideradas. Para un periodo concreto se podrá comparar el perfil de mejora en Andalucía con el de España. Si se consideran varios periodos se podrá ver la evolución regional de esta mejora.

\subsection{Direcciones de mejora}

Las direcciones de mejora se definirán de distinta forma según que la mejora de una variable venga determinada por un aumento o disminución de su valor.

Sea $x_{i t}$ el valor que toma una determinada variable para una región i en el momento t:

Si la mejora se produce al disminuir el valor de la variable se calcula

$$
D=\frac{\left(\min _{i} x_{i t+1}\right)}{\min _{i} x_{i t+1}-\max _{i}\left[x_{i t}: x_{i t+1}\right]}
$$

y su valor estandarizado en el gráfico radial viene definido por

$$
r_{i t}=1-\frac{\min _{i} x_{i t+1}-x_{i t}}{\min _{i} x_{i t+1}} * D
$$

$\mathrm{Si}$, por el contrario, un aumento del valor de la variable se corresponde con una mejora de esta, se calcula

$$
A=\frac{\left(\max _{i} x_{i t+1}\right)}{\max _{i} x_{i t+1}-\min _{i}\left[x_{i t}: x_{i t+1}\right]}
$$


y su valor estandarizado en el gráfico radial está definido por

$$
r_{i t}=1-\frac{\max _{i} x_{i t+1}-x_{i t}}{\max _{i} x_{i t+1}} * A
$$

De esta forma, para el periodo [t, $t+1$ ], la región i para la que mejor comportamiento haya tenido la variable en $\mathrm{t}+1$, tendrá un valor $r_{\mathrm{it}+1}=1$, todas las demás regiones tomarán un valor entre 0 y 1 para $\mathrm{t}+1$ (la demostración puede verse en el anexo 1), pudiéndose dar el caso de que en t tomase un valor mayor que 1 cuando la variable tome en $\mathrm{t}$ un valor mejor que el mejor valor alcanzado en $\mathrm{t}+1$. Por otra parte, la que peor comportamiento haya experimentado entre $t$ y $t+1$ tomará para ese año el valor 0 . Además, y por la forma en que se han estandarizado los valores, considerando como valores de referencia el mejor valor en $t+1$ y el peor entre $t$ y $t+1$, si $r_{\mathrm{it}+1}=0,5$, significará que la región i, para la variable considerada, ha mejorado entre t y $\mathrm{t}+1$ la mitad del rango.

\subsection{Indicador asociado a un objeto}

Para proporcionar una representación visual de la realidad en su conjunto, la figura 1 muestra un objeto formado por un polígono caracterizado por cuatro ejes que miden desde el centro 11,12,13 y 14 .

El área puede calcularse mediante la expresión de Schütz, Speckesser, y Schmid (1998):

$$
\text { Área }=((|1 *| 2)+(|2 *| 3)+(|3 *| 4)+(|4 *| 1)) * \text { sen (radianes 360/4) }
$$

Este valor puede utilizarse como indicador del objeto en sí, permitiendo su comparación con otros cuando únicamente la visualización no lo permita. Por ejemplo, en el caso de los dos objetos representado en la figura 2, su comparación visual es imposible, el indicador permitirá determinar cuál de ellos está mejor posicionado de forma global.

\section{DATOS DE ESTUDIO}

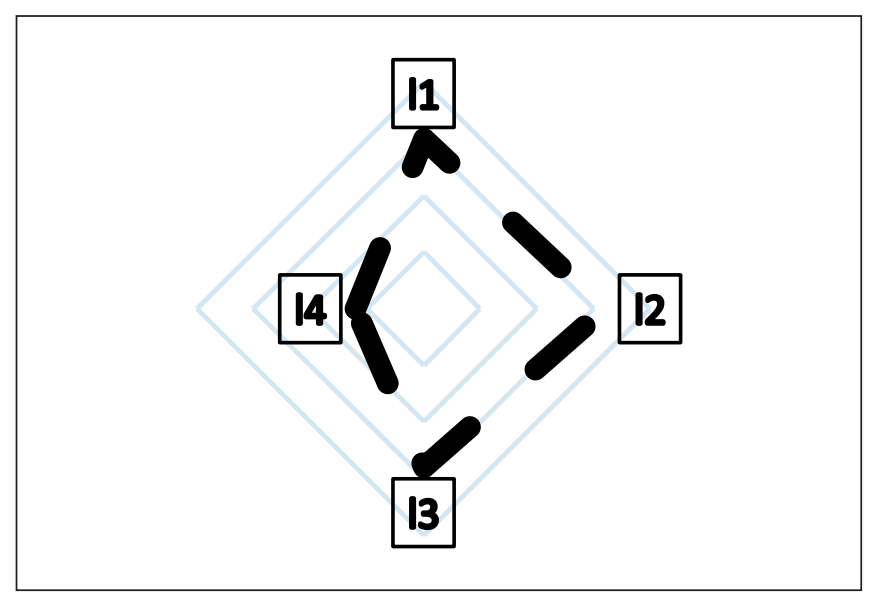

Figura 1. Representación en un gráfico radial de un objeto caracterizado por cuatro atributos.

Fuente: Elaboración propia.

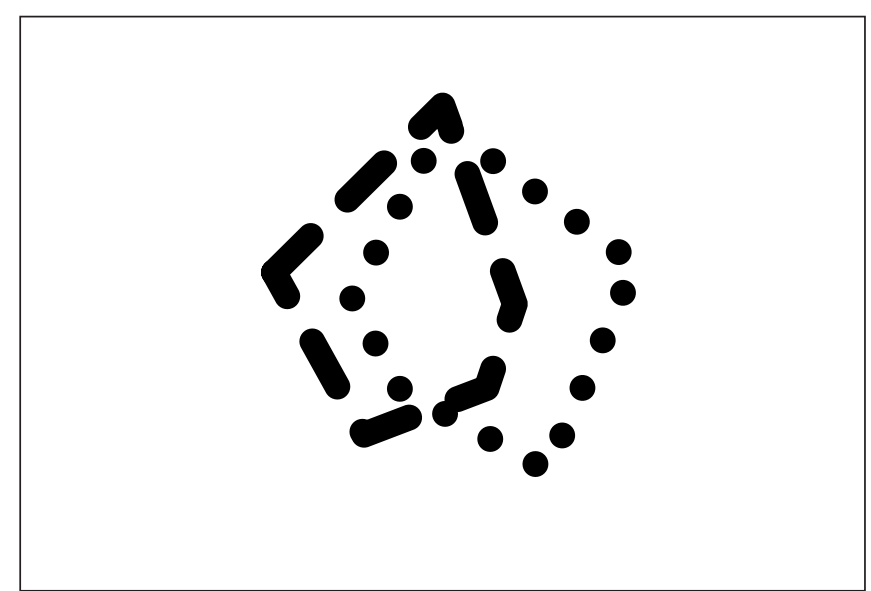

Figura 2. Representación en un gráfico radial de dos objetos caracterizados por cuatro atributos. Fuente: Elaboración propia.

\subsection{Variables}

Las definiciones de tasas esenciales que permiten evaluar el funcionamiento del sistema judicial consideradas y sus condiciones de mejora se recogen en la tabla 1. 
Tabla 1. Variables representativas del funcionamiento de la administración de justicia.

\begin{tabular}{|c|c|c|c|c|}
\hline Variable & Definición & & Significado & Dirección de mejora \\
\hline Tasa de litigiosidad & $\frac{n^{0} \text { asuntos ingresados en un año }}{n^{0} \text { de habitantes }}$ & *1000 & $\begin{array}{l}\text { Relaciona el número } \\
\text { de asuntos ingresados } \\
\text { en el sistema judicial } \\
\text { con el número de } \\
\text { habitantes }\end{array}$ & Disminución \\
\hline Tasa de resolución & $\begin{array}{l}n^{0} \text { asuntos resueltos en el año } \\
n^{0} \text { asuntos ingresados en el año }\end{array}$ & * 1000 & $\begin{array}{l}\text { Relaciona los asuntos } \\
\text { resueltos con el } \\
\text { número de asuntos } \\
\text { ingresados }\end{array}$ & Aumento \\
\hline Tasa de pendencia & $\frac{n^{\circ} \text { asuntos pendientes a final de año }}{n^{\circ} \text { asuntos resueltos en el año }}$ & $* 1000$ & $\begin{array}{l}\text { Relaciona los asuntos } \\
\text { pendientes con los } \\
\text { resueltos en un } \\
\text { mismo año }\end{array}$ & Disminución \\
\hline Tasa de congestión & $\frac{n^{0} \text { asuntos resueltos en el año }}{n^{\circ} \text { asun. ingres. }+n^{\circ} \text { asun. pend. princ. año }}$ & * 1000 & $\begin{array}{l}\text { Relaciona los asuntos } \\
\text { resueltos } \\
\text { con todos } \\
\text { los habría que tratar ese año }\end{array}$ & Disminución \\
\hline
\end{tabular}

Fuente: CGPJ. Elaboración propia.

\subsection{Datos}

Se recogen datos para Andalucía y se elaboran para el resto de España (España), calculando los correspondientes numeradores y denominadores de las tasas tomado las cifras que ofrece el CGPJ para España y restándole las correspondientes a Andalucía, para los años 2009, 2012, 2015, 2016 y 2018, con objeto de analizar las mejoras experimentadas entre los años 2009-2012, 2012-2015 y 2016-2018. Se presentan en la tabla 2.

Tabla 2. Datos originales sobre litigiosidad, resolución, pendencia y congestión. Años 2009, 2012, 2015,2016 y 2018.

\begin{tabular}{|c|c|c|c|c|c|}
\hline Tasa de Litigiosidad & 2009 & 2012 & 2015 & 2016 & 2018 \\
\hline Andalucía & 235,6 & 222,2 & 209,4 & 145,5 & 145,1 \\
\hline España & 197,8 & 182,6 & 173,1 & 120,2 & 124,5 \\
\hline Tasa de Resolución & 2009 & 2012 & 2015 & 2016 & 2018 \\
\hline Andalucía & 0,96 & 1,01 & 1,02 & 1,030 & 0,970 \\
\hline España & 0,96 & 1,02 & 1,03 & 1,036 & 0,959 \\
\hline Tasa de pendencia & 2009 & 2012 & 2015 & 2016 & 2018 \\
\hline Andalucía & 0,30 & 0,31 & 0,28 & 0,380 & 0,470 \\
\hline España & 0,35 & 0,32 & 0,28 & 0,368 & 0,446 \\
\hline Tasa de congestión & 2009 & 2012 & 2015 & 2016 & 2018 \\
\hline Andalucía & 1,31 & 1,30 & 1,27 & 1,370 & 1,460 \\
\hline España & 1,36 & 1,33 & 1,25 & 1,370 & 1,444 \\
\hline
\end{tabular}

Fuente: CGPJ. Elaboración propia. 


\section{RESULTADOS}

\subsection{Para todas las Jurisdicciones}

\subsubsection{Periodo: $2009-2012$}

En la tabla 3 se muestran las direcciones de mejora para el periodo 2009-2012. Se observa que Andalucía mejora su tasa de pendencia y congestión en mayor medida en lo que lo hace España y que empeora ostensiblemente en la tasa de litigios.

Representándolos en la figura 3, al tener Andalucía dos tasas mejorando y otras dos empeorando con respecto a España, es una situación típica en la que el gráfico no permite la visualización de la mejora conjunta de una respeto a la otra y debe acudirse al indicador global para concluir si Andalucía se ha situado mejor o peor que el resto de España. El Indicador para Andalucía en este periodo es de 1,225 mientras que para el resto está en 1,296. Dado que el máximo valor que puede tomar el indicador es 2, aunque ambas no están muy alejadas, lo está más Andalucía. Se deduce, pues que el funcionamiento de la Administración de Justicia en este periodo mejora menos en Andalucía.

Tabla 3. Direcciones de mejora para el periodo 2009-2012.

\begin{tabular}{|l|c|c|c|c|}
\hline \multicolumn{1}{l|}{} & litigiosidad & resolución & pendencia & congestión \\
\hline Andalucía & 0,252 & 0,956 & 1,000 & 1,000 \\
\hline España & 1,000 & 1,000 & 0,611 & 0,608 \\
\hline
\end{tabular}

Fuente: Elaboración propia.

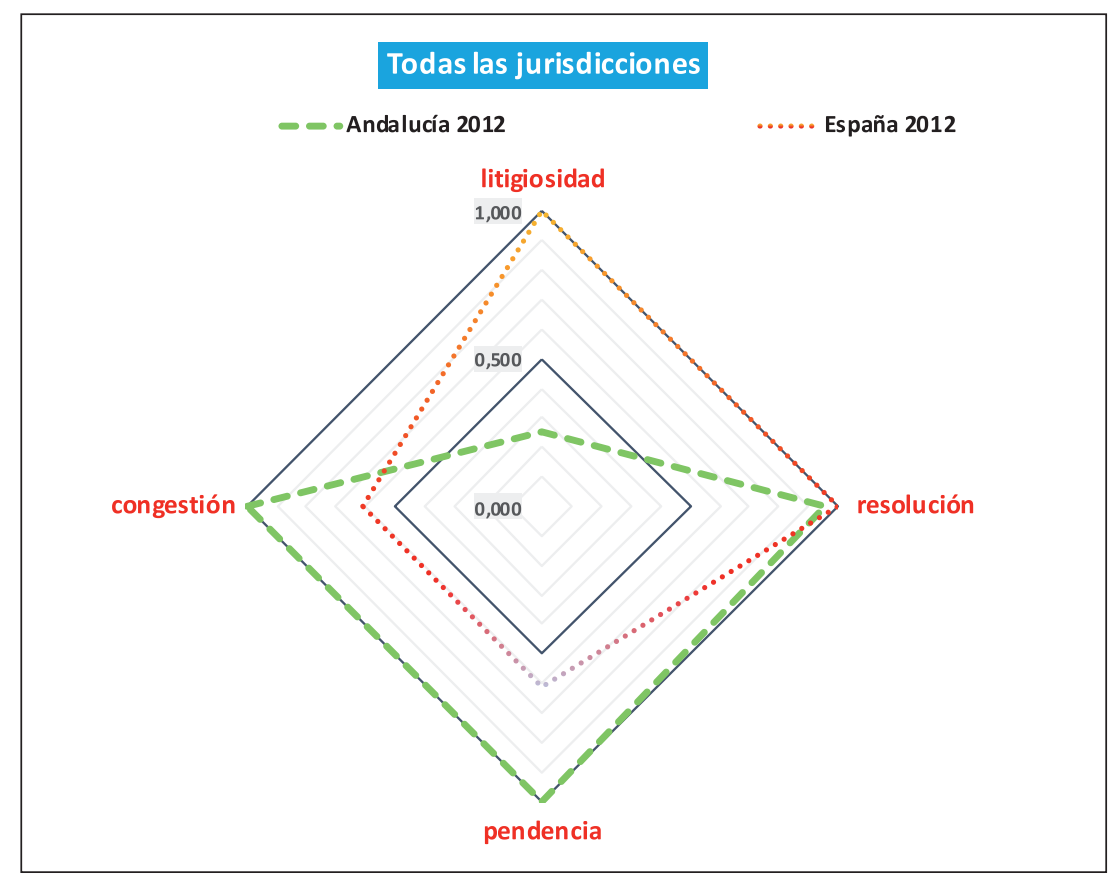

Figura 3. Posiciones de Andalucía y España en el espacio de mejora de 2009 a 2012.Fuente: Elaboración propia. 


\subsubsection{Periodo: $2012-2015$}

En la tabla 4 se muestran las direcciones de mejora para el periodo 2012-2015. En él se observa que Andalucía mantiene su tasa de pendencia como el resto de España, pero empeora en todas las demás. Esta circunstancia se aprecia en el gráfico radial (figura 4), en el que se visualiza un poliedro menor para Andalucía. El indicador toma los valores 0,754 y 2 para Andalucía y España respectivamente, dado que el aumento de la mejora experimentada en Andalucía por la tasa de pendencia no ha compensado el deterioro de las otras tasas.

España experimenta la máxima mejora en este periodo mientras que Andalucía se encuentra muy alejada, volviendo a destacar el dato negativo hallado para la litigiosidad.

Por último, resultando destacable, Andalucía empeora respeto al periodo 2009-2012, mientras que el resto mejora, implicando un aumento de la brecha entre ambas localizaciones geográficas. Si antes casi no existía diferencia en los indicadores, ahora supera a 1,2. Se tratará en mayor profundidad este punto en las tablas resúmenes.

Tabla 4. Direcciones de mejora para el periodo 2012-2015.

\begin{tabular}{|l|c|c|c|c|}
\cline { 2 - 5 } & litigiosidad & resolución & pendencia & congestión \\
\hline Andalucía & 0,261 & 0,572 & 0,966 & 0,656 \\
\hline España & 1,000 & 1,000 & 1,000 & 1,000 \\
\hline
\end{tabular}

Fuente: Elaboración propia.

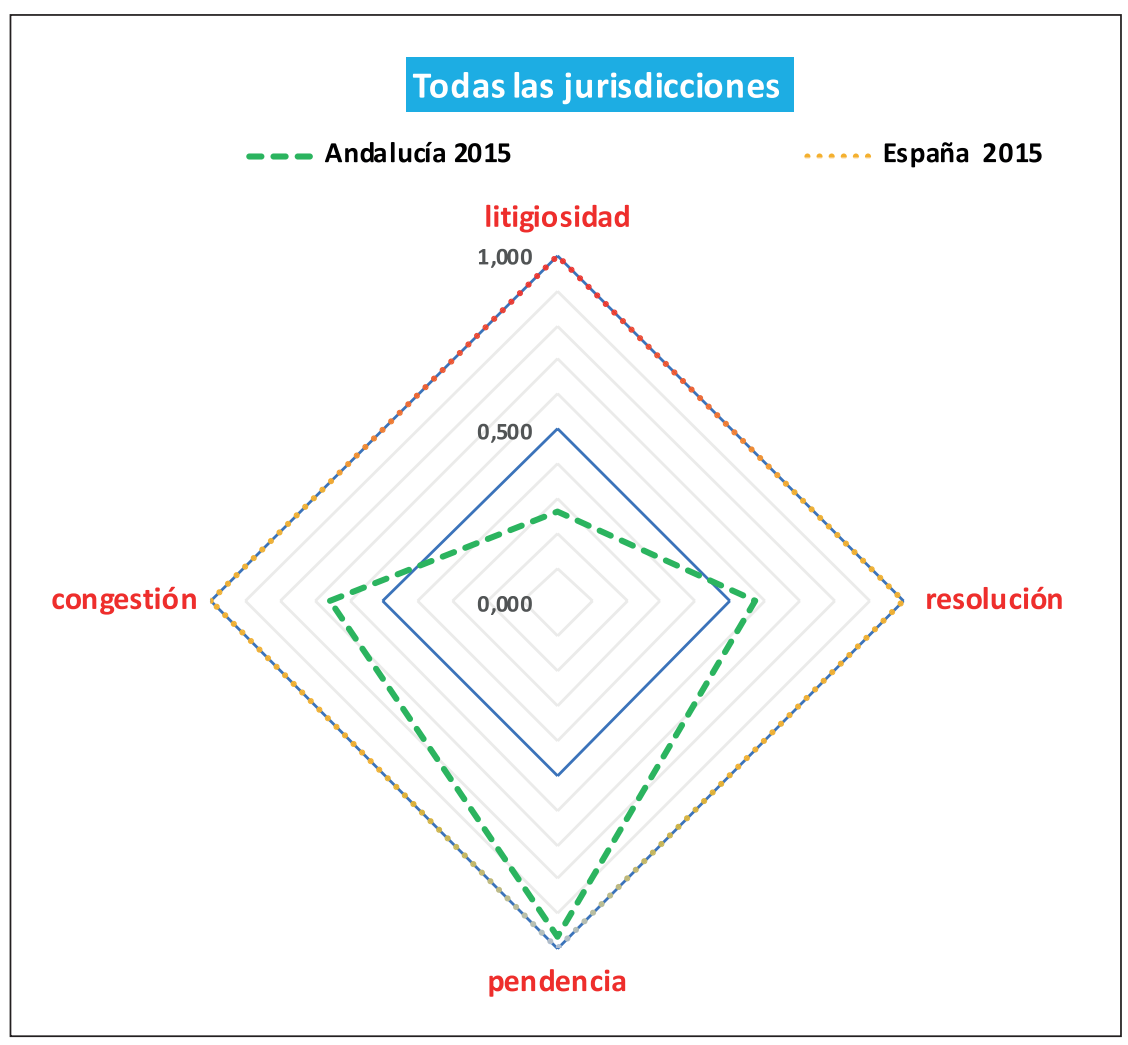

Figura 4. Posiciones de Andalucía y España en el espacio de mejoras de 2012 a 2015. Fuente: Elaboración propia. 


\subsubsection{Periodo: $2016-2018$}

En la tabla 5 se muestran las direcciones de mejora para el periodo 2016-2018. Este es un periodo especial en el que prácticamente se alcanzan los valores extremos siempre. Andalucía en la resolución, y España en el resto de las tasas. Por tanto, es el periodo en el que Andalucía se encuentra más perjudicada, como se aprecia en la figura 5 y se ratifica con los valores del indicador, de 0,011 para Andalucía y 1 para España, 0,989 puntos de diferencia entre ellos en perjuicio de Andalucía. Aun así, la distancia entre ambos ámbitos geográficos con respecto al periodo 2012-2015 se ha acortado, pero debido más a un empeoramiento de España que a una mejora de Andalucía.

Tabla 5. Direcciones de mejora para el periodo 2016 a 2018.

\begin{tabular}{|l|c|c|c|c|}
\cline { 2 - 5 } \multicolumn{1}{c|}{} & litigiosidad & resolución & pendencia & congestión \\
\hline Andalucía & 0,022 & 1,000 & 0,000 & 0,000 \\
\hline España & 1,000 & 0,000 & 1,000 & 1,000 \\
\hline
\end{tabular}

Fuente: Elaboración propia.

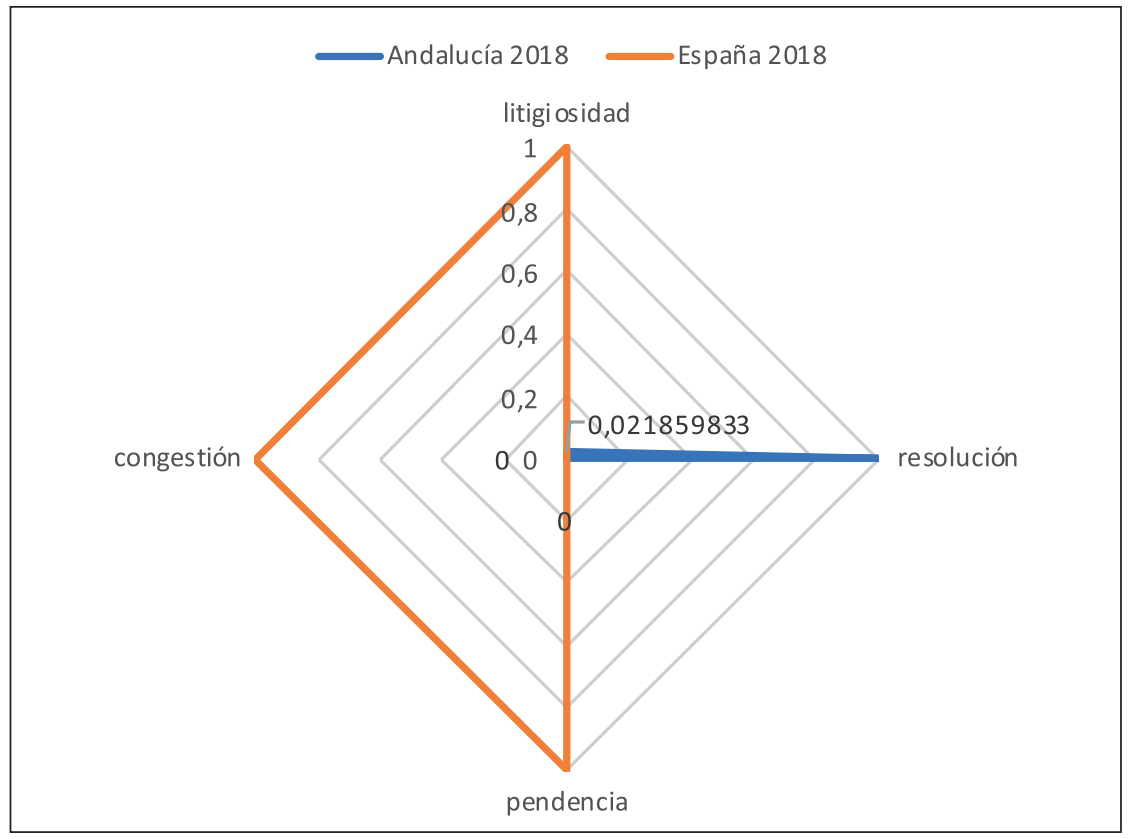

Figura 5. Posiciones de Andalucía y España en el espacio de mejora de 2016 a 2018. Fuente: Elaboración propia.

\subsubsection{Evolución de las mejoras}

En la figura 6 se recogen simultáneamente las posiciones de mejora para los entes regionales y en los periodos 2009-2012, 2012-2015 y 2016-2018. Visualmente no se aprecian las evoluciones por lo que se utilizan los indicadores globales que proporcionan una visión general de las distintas situaciones de mejora. En la figura 7 se representan sus valores, y se observa que la distancia entre Andalucía y España va aumentando considerablemente con el paso del tiempo, en detrimento de la situación de la Comunidad Autónoma de Andalucía. Es de destacar también la pérdida de posición en España del último periodo respecto al anterior, en la que el indicador cae hasta la mitad de su máximo valor. 


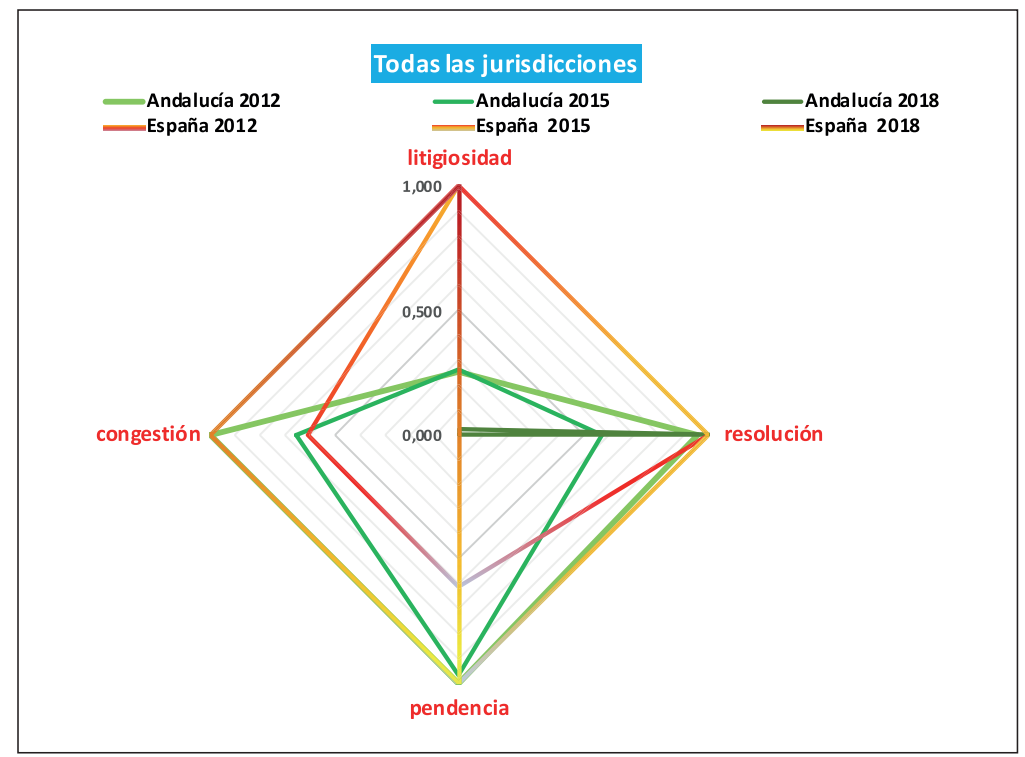

Figura 6. Posiciones de Andalucía y España en el espacio de mejoras. Fuente: Elaboración propia.

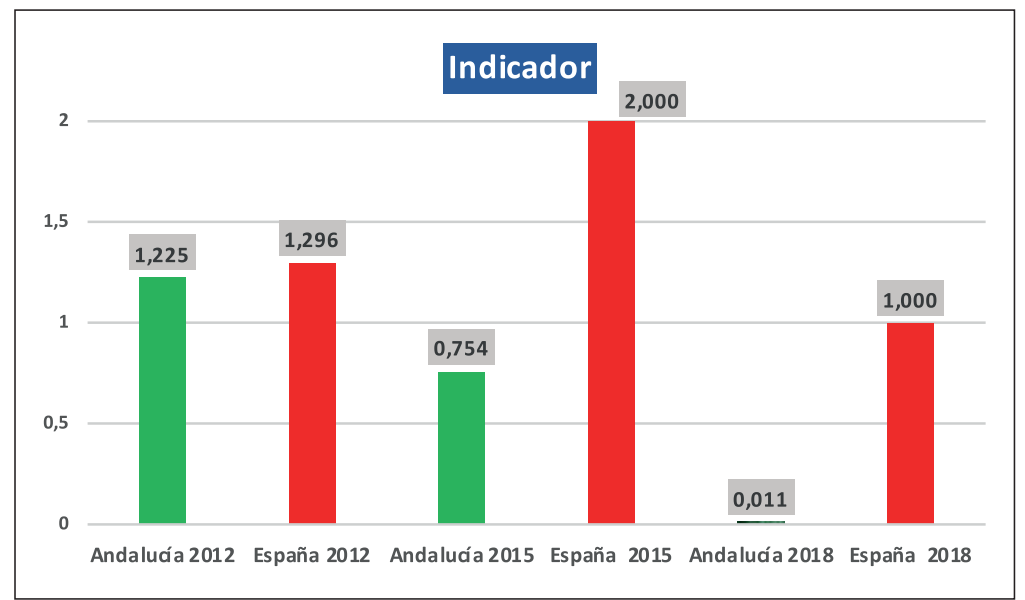

Figura 7. Indicadores globales. Fuente: Elaboración propia.

Para ver el alcance de este empeoramiento de la Comunidad de Andalucía, se ha repetido el análisis para cada una de las jurisdicciones.

\subsection{Resultados por jurisdicción}

Con objeto de hacer más cómoda la lectura, se expone una figura para cada una de las jurisdicciones. En cada una se presentan los gráficos radiales representativos de las mejoras experimentadas en los periodos considerados.

\subsubsection{Jurisdicción civil}

La figura 8 recoge las situaciones en la Jurisdicción civil, representando en cada fila el gráfico radial de cada subperiodo considerado. Para el periodo 2009-2012 se observa que, aun manteniendo en excelentes niveles sus tasas de litigiosidad y resolución, Andalucía empeora la congestión y la pendencia. La pendencia es un lastre que arrastra la Jurisdicción civil en Andalucía hasta llevarla a un valor del indicador en 1,29 puntos por debajo del de España, que, por otra parte, muestra un excelente comportamiento en el periodo.

Para el periodo 2012-2015, a la situación anterior de Andalucía se le suma una nula mejora en la resolución, lo que, sin embargo, no conduce a un mayor distanciamiento de España debido al empeoramiento de esta en los litigios. Como resultado se obtiene unos valores para el indicador de 0,340 y 1 respectivamente. 
En el último periodo, 2016-2018, se presenta una situación extrema, Andalucía no presenta mejora en ninguna de sus variables (todos son valores nulos) y, por el contrario, España alcanza los máximos valores en todas ellas (valores de 1 para la mejora de cada una)

\subsubsection{Jurisdicción penal}

La figura 9 recoge la situación en la Jurisdicción penal. En cada fila se representa el gráfico radial de cada subperiodo considerado. El periodo 2009-2012, presenta la cara opuesta a lo que ocurría en la jurisdicción civil, Andalucía mejora sus tasas de pendencia y congestión a pesar del marcado empeoramiento de la litigiosidad, haciendo que el indicador tome el valor 1,017, a 0,215 puntos de la mejora experimentada por España, lo que representa un 10,75\% del mejor valor que es un 2 .

El siguiente periodo, 2012-2015, continúa la tendencia del periodo anterior, empeorando menos la litigiosidad y más la resolución. Las situaciones comparadas son opuestas: donde España mejora su litigiosidad y resolución, Andalucía las empeora, y donde España empeora su pendencia y congestión, Andalucía presenta mejores valores. Como estas circunstancias se van compensando, se presenta aquí el caso de un indicador que toma valores muy próximos, 1,086 para Andalucía y 0,966 para España, escondiendo situaciones muy distintas.

En el último de los periodos analizados, 2016-2018, al igual que ocurría en la Jurisdicción civil, de nuevo se presenta una situación extrema, en la que Andalucía, salvo en la litigiosidad, alcanza los máximos valores de mejora. Así lo reflejan los indicadores globales: 1,336 para Andalucía y 0 para España.

\subsubsection{Jurisdicción contencioso-administrativa}

La figura 10 recoge la situación en la Jurisdicción contencioso-administrativa. Cada fila presenta el gráfico radial de cada subperiodo. Para el periodo 2009-2012, prácticamente coinciden las mejoras, aunque Andalucía está un poco mejor en su tasa de resolución, y peor en la de litigios. Como resultado se obtienen respectivamente los valores 1,891 y 1,427 para el indicador.

El periodo 2012-2015 presenta el límite de la situación del periodo anterior, con distancias máximas entre la resolución, a favor de Andalucía, y la litigiosidad, en favor de España. Los valores del indicador quedan, así, compensados, con valores respectivos de 0,962 y 0,893, muy próximos, pero correspondientes a situaciones muy distintas.

En el periodo 2016-2018 se produce un cambio total en la tendencia: España alcanza sus valores máximos y Andalucía los mínimos, salvo en la litigiosidad que resulta un poco menos perjudicada.

\subsubsection{Jurisdicción social}

La figura 11 recoge la situación en la Jurisdicción social. En cada fila se presenta el gráfico radial del subperiodo. Para el periodo 2009-2012, esta es la única jurisdicción en la que Andalucía destaca por su buen comportamiento en la tasa de litigios, en detrimento de su pendencia y congestión. Con valores para el indicador de 0,136 y 1,433, para Andalucía y España, respectivamente, Andalucía tiene una diferencia de 1,297 con España.

En el periodo 2012-2015 se repite la situación del periodo anterior, aunque se disminuyen las distancias entre la litigiosidad y la resolución, la primera en favor de España y la segunda en favor de Andalucía. El indicador toma los valores 0,349 y 1,616, respectivamente, manteniendo, así, entre ellos, la misma diferencia que en el periodo 2009-2012.

Andalucía continúa con su máxima mejora en la litigiosidad en el periodo 2016-2018, mientras que el resto de los valores no muestran mejora alguna. Para España, por el contrario, en este periodo su mejora es nula en la litigiosidad y máxima en el resto de las variables. Así, el indicador toma los valores respectivos 0 y 1.

\subsubsection{Evolución a través de los indicadores globales}

A modo de resumen, se muestra la evolución de cada jurisdicción mediante los valores del indicador, aunque, como ya se ha mencionado anteriormente, a veces este oculta situaciones muy distintas, ya que puede quedar compensada la mejora en una de las variables con el empeoramiento en otra (en el anexo 2 se presentan los gráficos de los indicadores para cada uno de los periodos considerados como forma alternativa de comparación). 


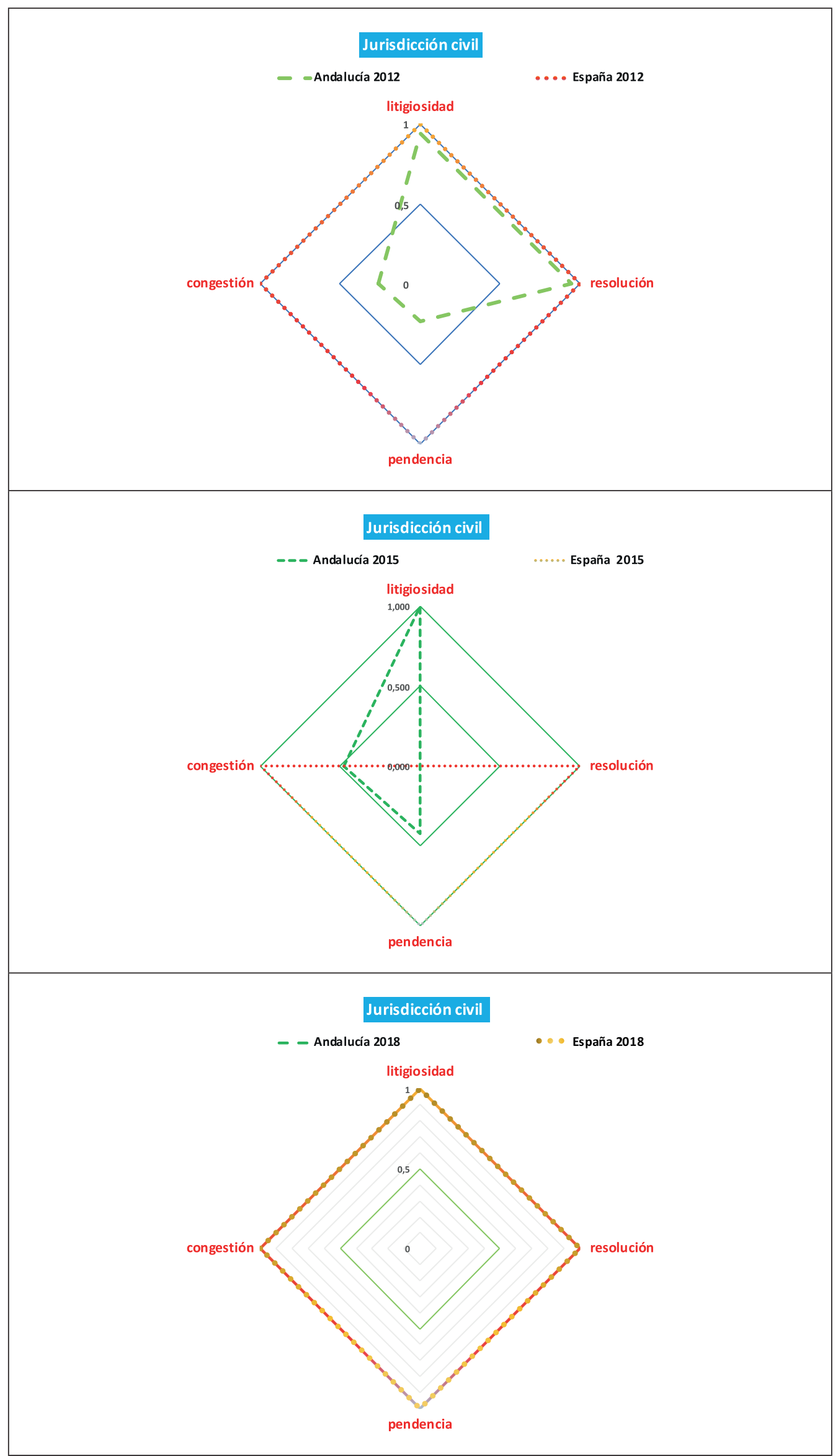

Figura 8. Jurisdicción civil. Gráficos radiales de las mejoras en los periodos 2009-2012, 2012-2015 y 2016-2018. Fuente: Elaboración propia. 


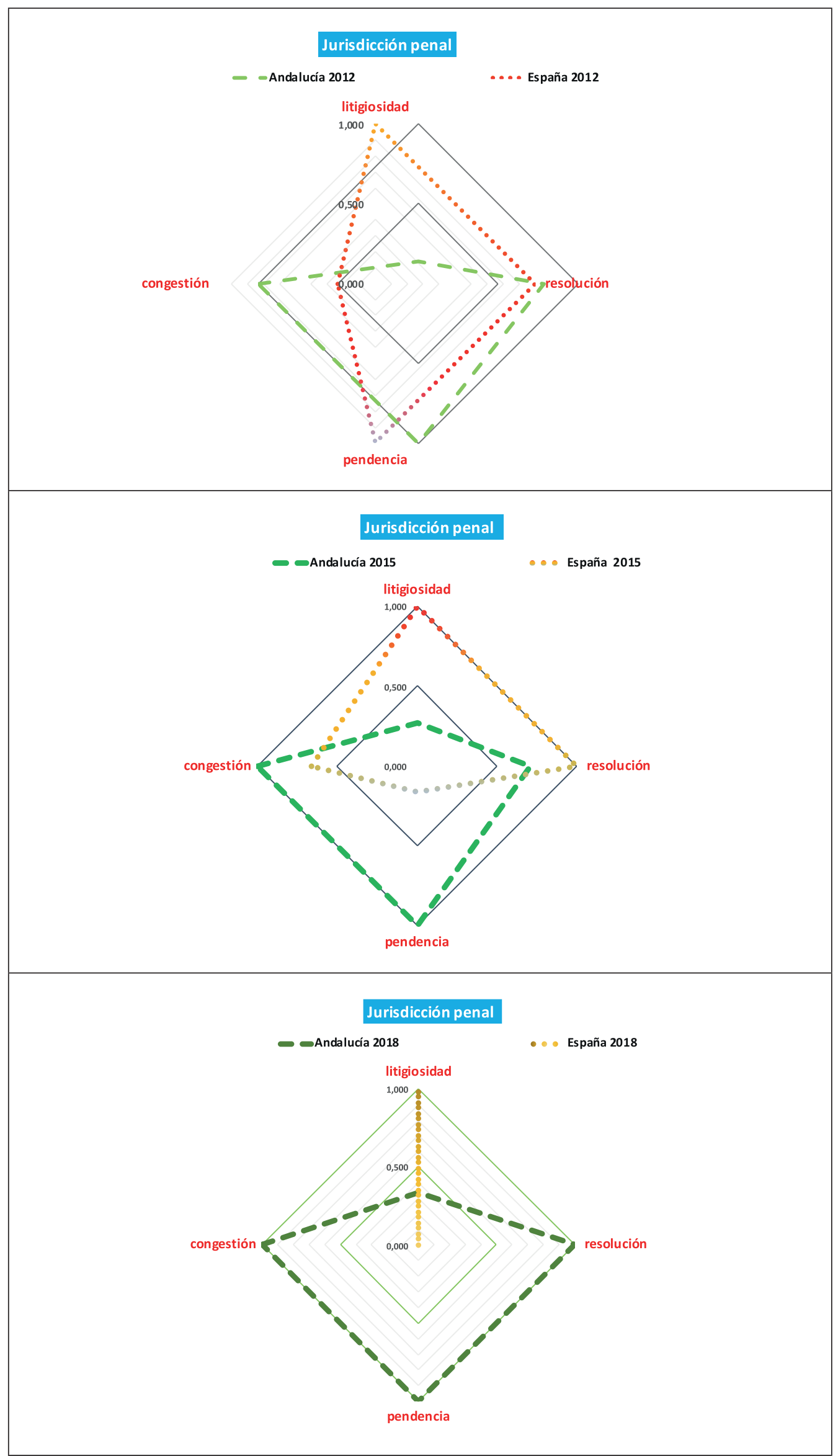

Figura 9. Jurisdicción penal. Gráficos radiales de las mejoras en los periodos 2009-2012, 2012-2015 y 2016-2018. Fuente: Elaboración propia. 


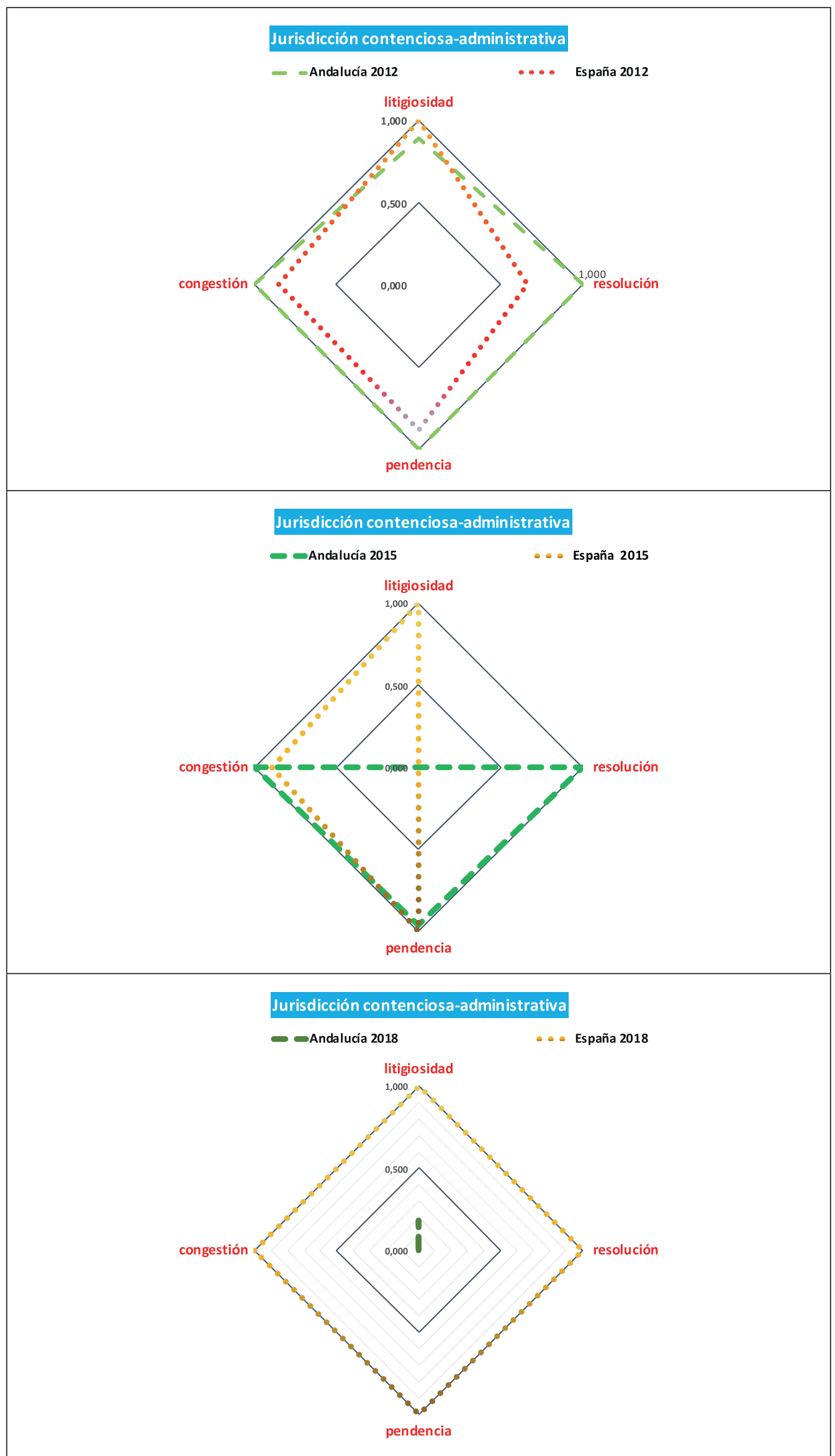

Figura 10. Jurisdicción contencioso-administrativa. Gráficos radiales de las mejoras en los periodos 2009-2012, 2012-2015 y 2016-2018. Fuente: Elaboración propia. 


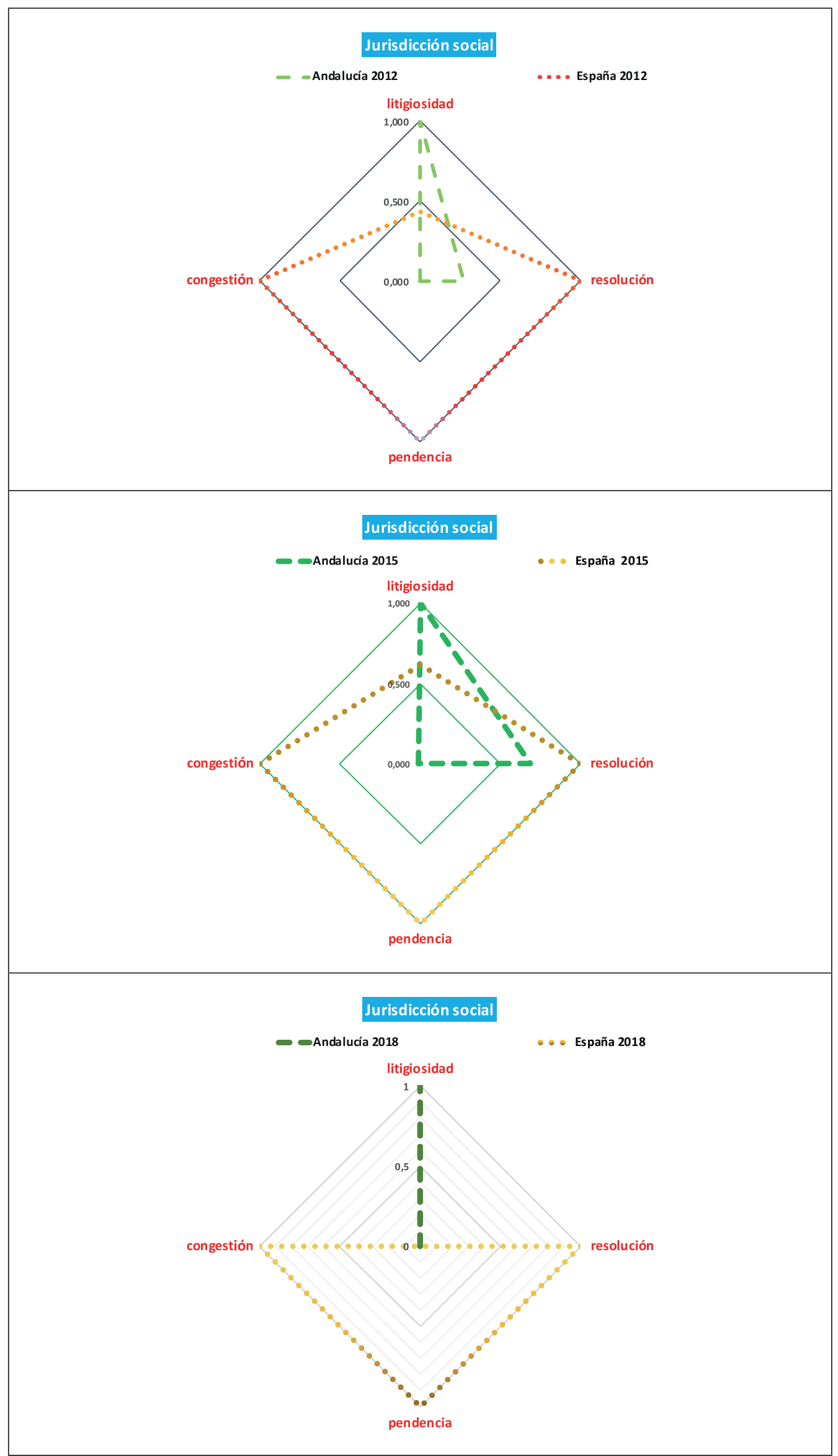

Figura 11. Jurisdicción social. Gráficos radiales de las mejoras en los periodos 2009-2012, 2012-2015 y 2016-2018. Fuente: Elaboración propia. 
Para la jurisdicción civil se obtienen unos perfiles, figura 12, para Andalucía tan alejados de los de España que van a repercutir negativamente en el comportamiento de todas las jurisdicciones, representados en la figura 7. Sin embargo, los resultados en la jurisdicción penal, figura 13, repercuten positivamente en todas las jurisdicciones pues, para ellos, Andalucía, aunque ligeramente inferior en el primer periodo considerado, 2009-2012, en el periodo siguiente 2012-2015, se sitúa por encima de España, para terminar en el periodo 2016-2018 distanciándose de ella, de manera positiva, considerablemente.

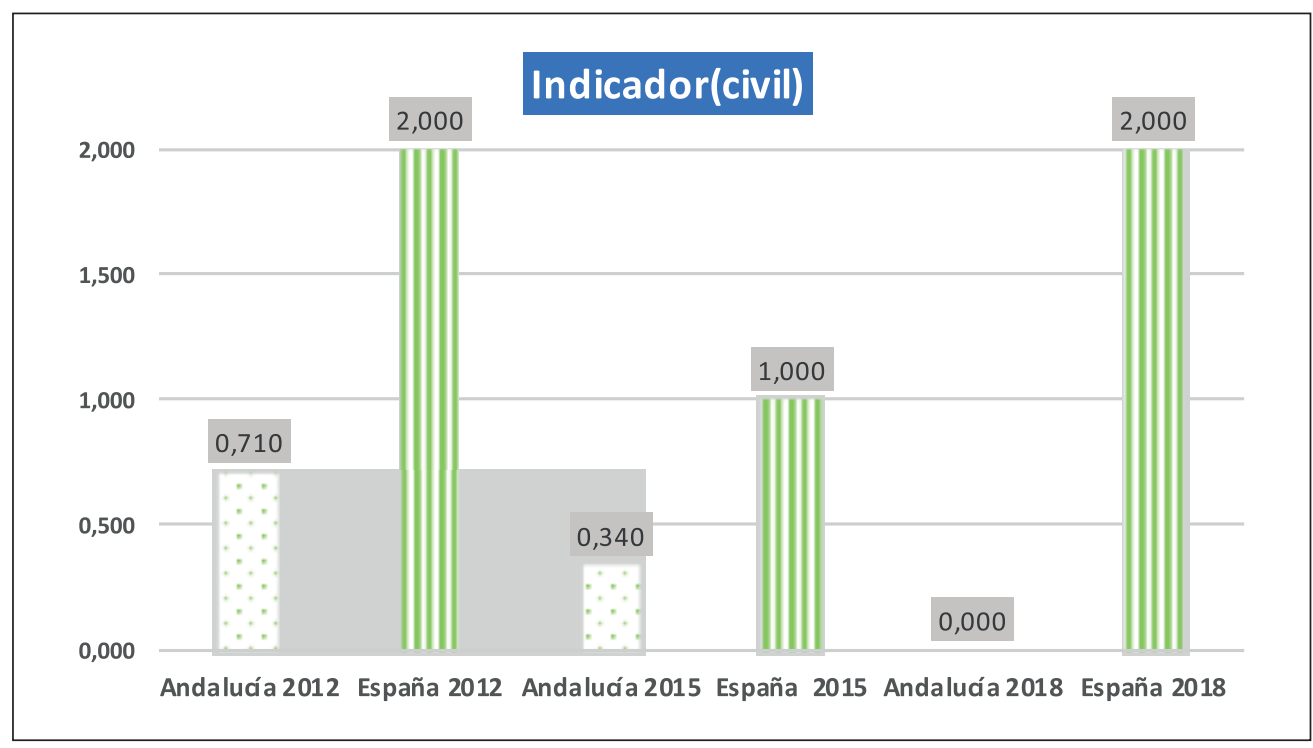

Figura 12. Indicadores de mejoras en la jurisdicción civil. Fuente: Elaboración propia.

En la Jurisdicción contenciosa-administrativa ocurre todo lo contrario que en la jurisdicción penal (figura 14). Andalucía comienza con una notable mejora respecto a España que va perdiendo con el tiempo, hasta llegar a distanciarse de manera negativa lo máximo posible. Esta jurisdicción, por tanto, también a influir negativamente en los perfiles de la figura 7.

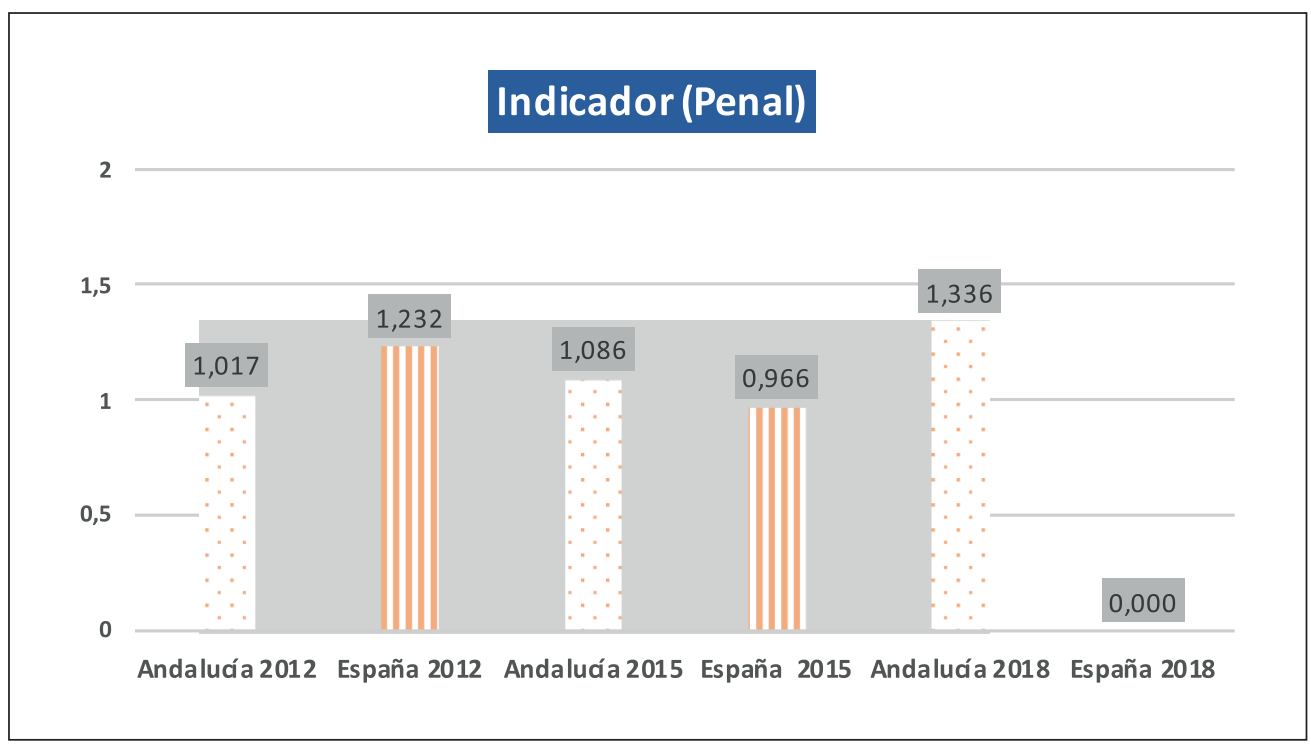

Figura 13. Indicadores de mejoras en la jurisdicción penal.

Fuente: Elaboración propia. 


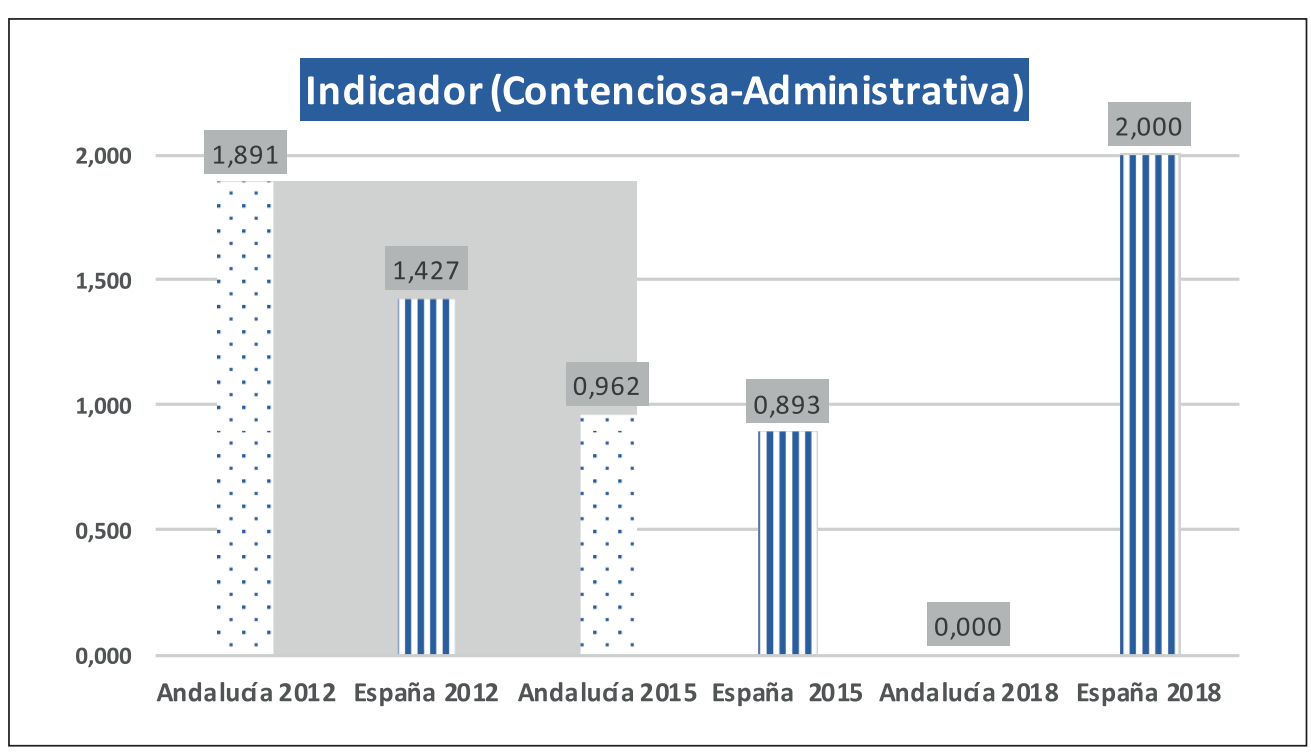

Figura 14. Indicadores de mejoras en la jurisdicción contenciosa-administrativa. Fuente: Elaboración propia.

El comportamiento en la jurisdicción social, figura 15, es análogo al de la jurisdicción civil: Andalucía se mantiene en todos los periodos muy por debajo de las mejoras de España, aunque las distancias son menores que en la jurisdicción civil, en la que coincide con su perfil.

En definitiva, podemos concluir que son las jurisdicciones civil y social las que fundamentalmente presentan peores comportamientos en Andalucía respecto a España y arrastran en este comportamiento a los resultados obtenidos para todas las jurisdicciones.

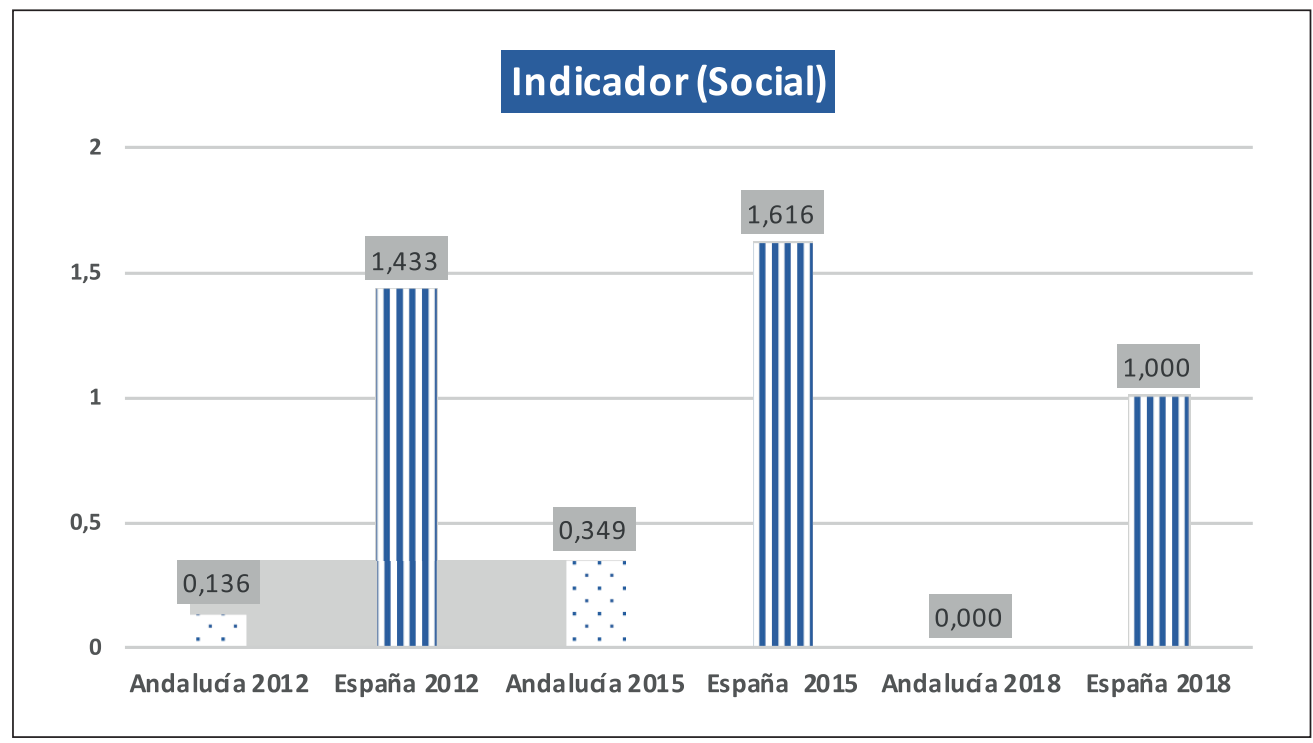

Figura 15. Indicadores de mejoras en la jurisdicción social.Fuente: Elaboración propia.

\section{DISCUSIÓN Y CONCLUSIONES}

En España, tal como se encuentra diseñado el sistema judicial, con parte de sus competencias transferidas a sus CCAA, no se puede analizar con propiedad del funcionamiento de su sistema judicial si no es a través del funcionamiento de cada uno de los sistemas judiciales que lo componen. Las CCAA destinan a este sector pú- 
blico cantidades que pueden oscilar desde los 4.565.958,29 euros que destina Andalucía por cada 100.000 habitantes hasta incluso los 8.529.186,88 euros por cada 100.000 habitantes que destina La Rioja (datos extraídos de los presupuestos de las CCAA correspondientes a 2015). También el número de jueces y fiscales varía ostensiblemente entre las CCAA, así como el número de asuntos ingresados, haciendo que, por ejemplo, en Andalucía haya 1.881 asuntos ingresados por cada juez, mientras que en La Rioja esta cifra baja hasta los 1.043 (datos del CGPJ correspondientes a 2015). Estas diferencias regionales son persistentes en el tiempo (Gutiérrez-López, 2016). Ahora bien, ¿a pesar de estas diferencias, las potenciales mejoras en su funcionamiento siguen la misma dirección o también en ellas se detectan divergencias regionales?. El trabajo aborda esta cuestión considerando simultáneamente los comportamientos de las tasas de litigiosidad, resolución, pendencia y congestión, que, según el CGPJ, son las tasas representativas del funcionamiento de una Administración de Justicia. El propio Consejo calcula las variaciones anuales de estas tasas, y las analiza y compara entre las distintas CCAA en sus publicaciones anuales "La Justicia dato a dato "y "Panorámica de la Justicia" respectivamente, pero tratadas de forma individual, lo que no proporciona una visión global del funcionamiento. La literatura académica especializada considera la duración de los procedimientos (medida a través de la tasa de congestión), como la medida más relevante y completa del funcionamiento de una Administración de Justicia (MoraSanguinetti y Garoupa, 2015), en concreto la correspondiente a la jurisdicción civil, por ser esta la que tiene más asuntos ingresados, pero esta consideración carece de toda fundamentación teórica. Es, por tanto, este trabajo un tratamiento pionero en la consideración simultánea de las cuatro principales tasas para el análisis de un sistema judicial.

Por otra parte, la metodología de los gráficos radiales, que, aplicada a sucesivos periodos, permite visualizar la evolución de un sistema, y que ha sido usada en el análisis de otros sectores, como la educación o el mercado laboral, no ha sido, a conocimiento de los autores, aplicada al sistema judicial. Esto es de por sí un valor añadido del trabajo ya que, entre otras ventajas, permite de manera muy visual deducir si las escasas medidas que se vienen adoptando hacen que los sistemas judiciales de las distintas CCAA vayan convergiendo o no. En este trabajo se aplica a Andalucía y al resto de España, ambas consideradas como dos regiones independientes, y a las mejoras experimentadas en tres periodos consecutivos, 2009-2012, 2012-2015 y 2016-2018. Se observa cómo las distancias entre ellas van aumentando, y no solo en lo relativo a todas las jurisdicciones, sino que, incluso si se analiza por jurisdicción, solo la penal y la contenciosa-administrativa mantienen un comportamiento convergente, que queda absorbido por los malos resultados para las jurisdicciones civil y social cuando se consideran conjuntamente.

La principal debilidad que presenta la metodología de los gráficos radiales es que los resultados que con ella se obtienen son muy sensibles a las variables que se consideren como representativas del objeto analizado. En el presente estudio se soslaya esta debilidad utilizando, para representar el sistema judicial de una región, las tasas que, como se ha mencionado anteriormente, el propio CGPJ considera esenciales para analizar el funcionamiento de un sistema judicial.

Por otra parte, aunque la técnica proporciona solo una fotografía de la situación, sin entrar en consideraciones de cuáles son las circunstancias que han llevado a la misma, e imposibilitando que a través de ella se puedan extraer conclusiones acerca del porqué de la convergencia o no en las distintas Jurisdicciones, (en los trabajos previos de Váquez-Cueto y Gutiérrez-López (2017), se contrastan si determinadas variables pueden haber conducido a esta situación), sí que proporciona una visión de lo que ocurre y, con ello, una línea de actuación consistente en mejorar aquella dimensión (tasa) que hace que una región se descuelgue de la mejora experimentada por otra.

En definitiva, el trabajo presenta una metodología susceptible de ser aplicada a un sector público tan importante para un país como lo es su Justicia.

\section{REFERENCIAS}

Aldunce, P., Neri, C. \& Szlafsztein, C. (2008). Hacia la evaluación de prácticas de adaptación ante La variabilidad y el cambio climático. Editado por Szlafsztein. Belém. NUMA/UFPA.

Bazzani, T, De le Court, A., Diveeva, N., Eleveld, A., Klawitter, S., Singer, R.,... Sychenko, E. (2018). Dealing with Unemployment. Labour Market Policy Trends Editors. Tania Bazzani, Reinhard Singer Humboldt-Universität zu Berlin. Faculty of Law. 
Centro de Investigaciones Sociológicas. Recuperado de www.cis.es

Consejo General Del Poder Judicial. La Justicia dato a dato. Recuperado de www.poderjudicial.es

Consejo General Del Poder Judicial. Panorámica de la Justicia. Recuperado de www.poderjudicial.es

Gutiérrez-López, F. (2016). Gasto Público y funcionamiento de la Justicia en España entre 2004 Y 2013: Especial referencia a la Comunidad Autónoma de Andalucía. Comparativa con otros países europeos. (Tesis doctoral). Universidad de Sevilla, Facultad de Ciencias Económicas y Empresariales, España. Recuperado de http://hdl.handle.net/11441/39799.

Instituto Nacional de Estadística. Cifras del padrón de habitantes. Recuperado de www.ine.es

Moerke, A. (1997). Does governance matter? Performance and corporate governance structures of Japanese keiretsu groups. WZB Discussion Paper, N. FS IV, 97-43. Berlin. Recuperado de http://hdl.handle.net/10419/51173

MoraSanguinetti, J. S. \& Garoupa, N. (2015). Do lawyers induce litigation? Evidence from Spain, 20012010. International Review of Law and Economics, 44, 2941. https://doi.org/10.1016/j.irle.2015.06.003

Mosley, H.;, Mayer, A. (1999). Benchmarking national labour market performance:. a radar chart approach. WZB Discussion Paper, №. FS I 99-202, Berlín. Recuperado de http://hdl.handle.net/10419/43952

Pastor, S. (2003): Dilación, eficiencia y costes : Foro sobre la Reforma y Gestión de la Justicia, . Documento de trabajo n5, Fundación BBVA, Bilbao. Recuperado de http://www.fbbva.es

Pastor, S.; Rosales, V. (2005): Dos dimensiones de la eficacia de la justicia. Economistas, n. ${ }^{\circ}$ 105, pp. $103-114$.

Pérez Benedito, M. A.; Ángel, M. (2011): Análisis de estrategias de gestión: aplicaciones polivalentes del gráfico radial. Revista iberoamericana de contabilidad de gestión RIGC IX, Vol. IX · n 17, pp. 1-34.

Pérez Benedito, M.A. (2013). Aplicación de los gráficos radiales en la evaluación del servicio público de transportes. Auditoría Pública, 60, 73-96.

Pérez Benedito, M. Á. (2016). La aplicación de los gráficos radiales en el análisis contable del sector pesquero en Cataluña: Gráfico radial y análisis contable. Revista Internacional de Economía y Gestión de Las Organizaciones, 1 (2), 99-128.

Schütz, H., Speckesser, S. \& Schmid, G. (1998). Benchmarking labour market performance and labour market policies: Theoretical foundations and applications. Discussion Paper, No. FS I, 98-205, (WZB), Berlin. Recuperado de http:// hdl.handle.net/10419/43918

Vázquez Cueto, M. J. \& Gutiérrez López, F. (2017). ¿Está justificada la mala imagen de la administración de justicia española? ¿Es un problema de inversión?: Una comparativa europea mediante el análisis DEA. Revista De Estudios Empresariales. Segunda Época, 2. https://doi.org/10.17561/ree.v0i1.3190

Vázquez Cueto, M.J. \& Gutiérrez López, F. (2017). Gasto público, medios personales y funcionamiento de la Administración de Justicia en España: ¿el problema es el gasto? Indret: Revista para el Análisis del Derecho, (4), 6. 


\section{Anexo 1}

Si la dirección de mejora se produce al disminuir el valor de la variable y $x_{i t+1}=\left(\min _{i} x_{i t+1}\right)$,entonces

$$
r_{i t+1}=1-\frac{\min _{i} x_{i t+1}-x_{i t+1}}{\min _{i} x_{i t+1}} * D=1-0 * D=1
$$

Y para el caso extremo en que $x_{i t+1}=\left(\max _{i} x_{i t+1}\right)$, entonces

$$
r_{i t+1}=1-\frac{\min _{i} x_{i t+1}-\max _{i} x_{i t+1}}{\min _{i} x_{i t+1}} * \frac{\left(\min _{i} x_{i t+1}\right)}{\min _{i} x_{i t+1}-\max _{i}\left[x_{i t}: x_{i t+1}\right]}=1-\frac{\min _{i} x_{i t+1}-\max _{i} x_{i t+1}}{\min _{i} x_{i t+1}-\max _{i}\left[x_{i t}: x_{i t+1}\right]}=
$$

$\frac{\max _{i} x_{i t+1}-\max _{i}\left[x_{i t}: x_{i t+1}\right]}{\min _{i} x_{i t+1}-\max _{i}\left[x_{i t}: x_{i t+1}\right]}<1$, pues si fuese mayor o igual a 1 ocurriría que

$\max _{i} x_{i t+1}-\max _{i}\left[x_{i t}: x_{i t+1}\right] \geq \min _{i} x_{i t+1}-\max _{i}\left[x_{i t}: x_{i t+1}\right] \leftrightarrow \max _{i} x_{i t+1} \leq \min _{i} x_{i t+1}$, que no tiene sentido, pero no puede ser menor que 0 , pues esto ocurriría si

$$
\frac{\min _{i} x_{i t+1}-\max _{i} x_{i t+1}}{\min _{i} x_{i t+1}-\max _{i}\left[x_{i t}: x_{i t+1}\right]}>1
$$

Lo que implicaría que $\min _{i} x_{i t+1}-\max _{i} x_{i t+1}>\min _{i} x_{i t+1}-\max _{i}\left[x_{i t}: x_{i t+1}\right] \leftrightarrow, \max _{i} x_{i t+1}<\max _{i}\left[x_{i t}: x_{i t+1}\right]$, que no tiene sentido.

Si la dirección de mejora se produce cuando la variable aumenta y $x_{i t+1}=\left(\max _{i} x_{i t+1}\right)$, entonces

$$
r_{i t+1}=1-\frac{\max _{i} x_{i t+1}-x_{i t+1}}{\max _{i} x_{i t+1}} * A=1-0 * A=1
$$

Y para cualquier otro valor $x_{i t+1}<\left(\max _{i} x_{i t+1}\right)$, por ejemplo, el caso extremo en que $x_{i t+1}=\min _{i} x_{i t+1}$, entonces

$$
\begin{gathered}
r_{i t+1}=1-\frac{\max _{i t+1}-\min _{i} x_{i t+1}}{\max _{i} x_{i t+1}} * \frac{\max _{i} x_{i t+1}}{\max _{i} x_{i t+1}-\min _{i}\left[x_{i t}: x_{i t+1}\right]} \\
=1-\frac{\max _{i} x_{i t+1}-\min _{i} x_{i t+1}}{\max _{i} x_{i t+1}-\min _{i}\left[x_{i t}: x_{i t+1}\right]}<1
\end{gathered}
$$

Puesto que $\min _{i}\left[x_{i t}: x_{i t+1}\right]<\min _{i} x_{i t+1}<\max _{i} x_{i t+1}$, tanto el numerador como el denominador son positivos $\mathrm{y}$, por tanto, el cociente es positivo y el resultado es $<1$.

Además, no puede ser menor que cero pues esto significaría que

$$
\frac{\max _{i} x_{i t+1}-\min _{i} x_{i t+1}}{\max _{i} x_{i t+1}-\min _{i}\left[x_{i t}: x_{i t+1}\right]}>1 \leftrightarrow \max _{i} x_{i t+1}-\min _{i} x_{i t+1}>\max _{i} x_{i t+1}-\min _{i}\left[x_{i t}: x_{i t+1}\right] \leftrightarrow \min _{i} x_{i t+1}<\min _{i}\left[x_{i t}: x_{i t+1}\right],
$$

que no tiene sentido.

Queda así demostrado que todos los valores que reflejan la mejora experimentada en $\mathrm{t}+1$ respecto a $\mathrm{t}$ toman valores entre 0 y 1 . 


\section{Anexo 2: Indicadores de las distintas jurisdicciones por periodo.}

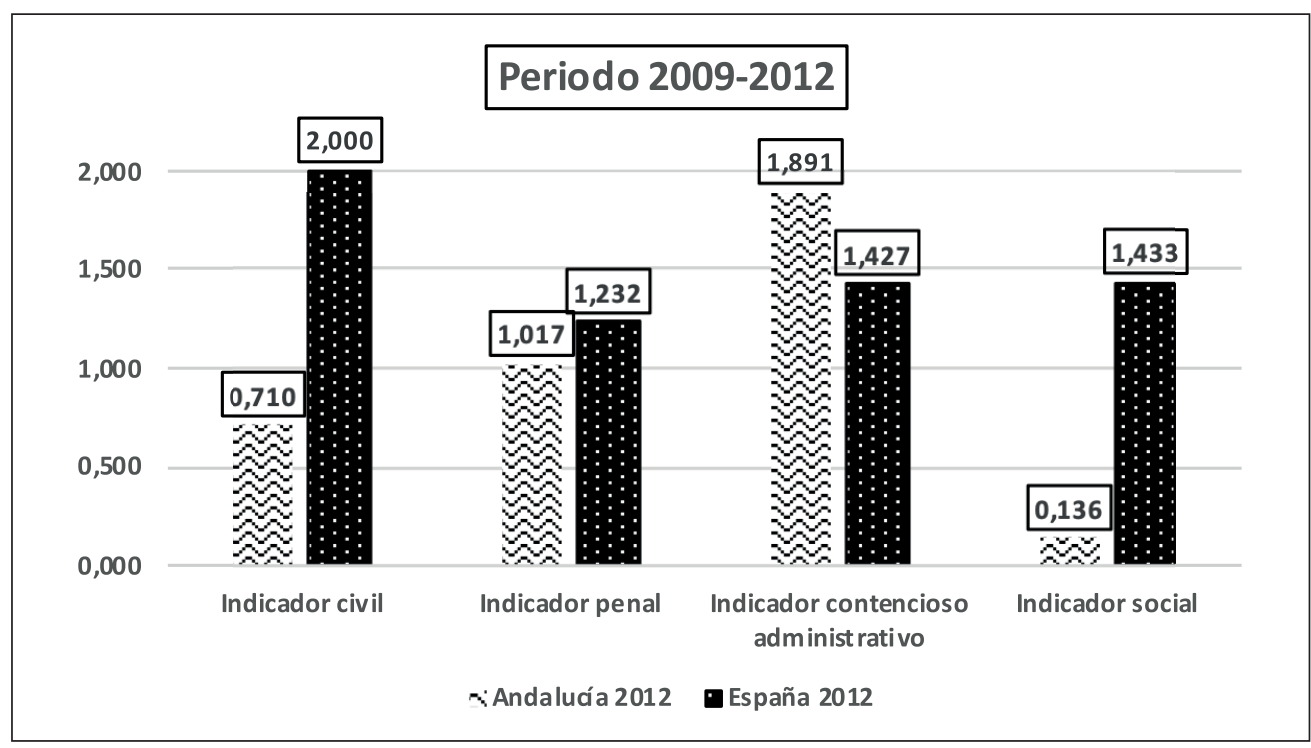

Fuente: Elaboración propia. Fuente de datos: La Justicia dato a dato. CGPJ.

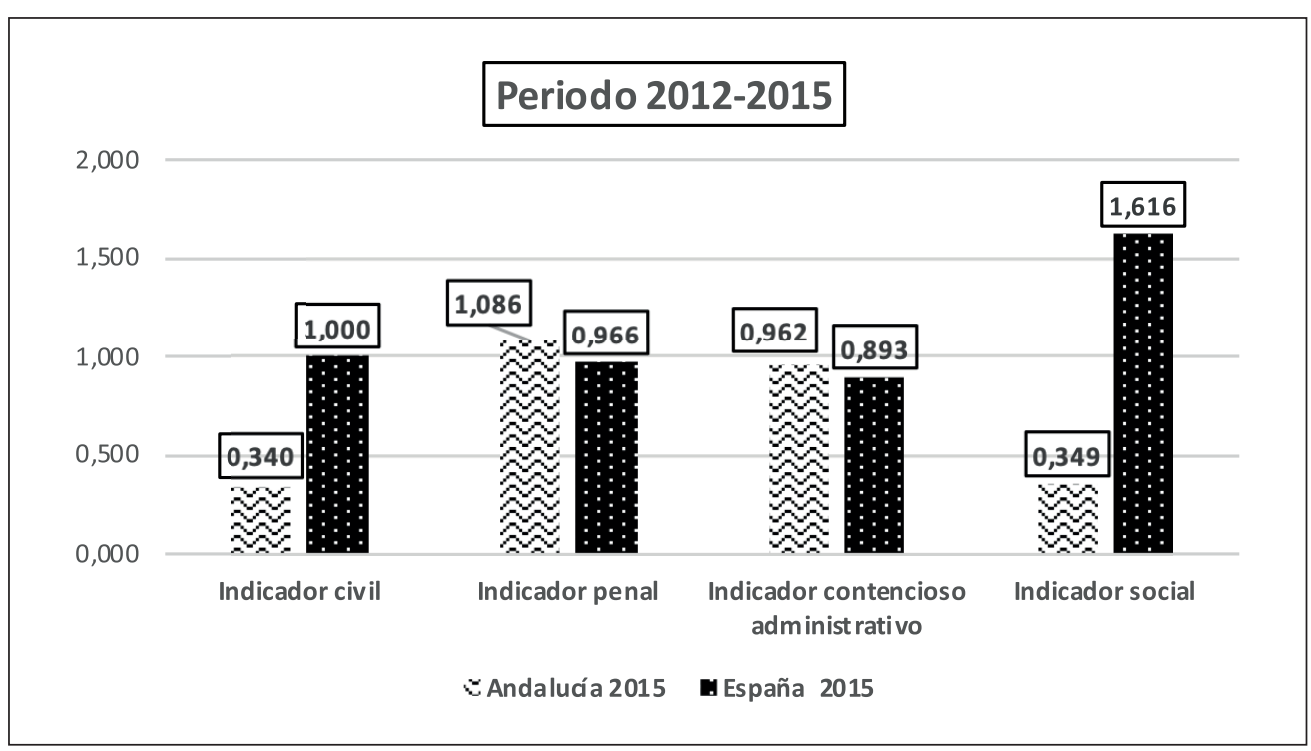

Fuente: Elaboración propia. Fuente de datos: La Justicia dato a dato. CGPJ. 


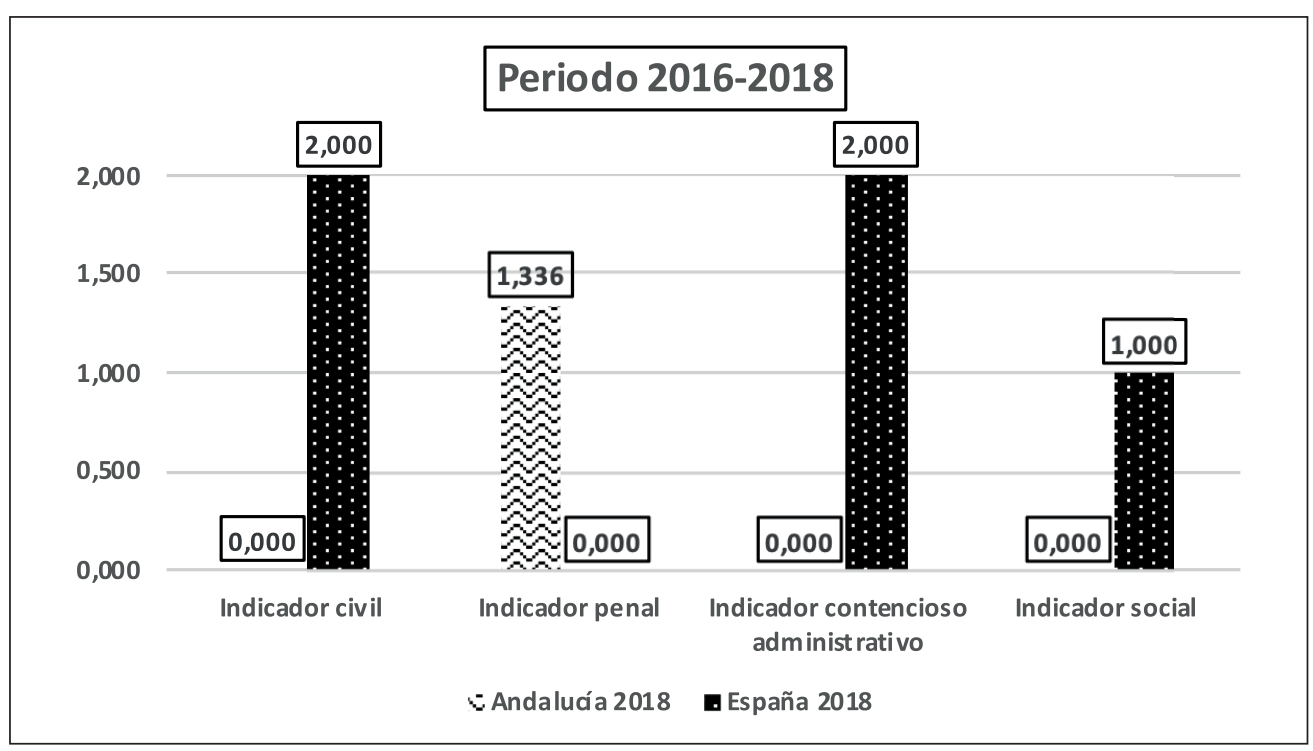

Fuente: Elaboración propia. Fuente de datos: La Justicia dato a dato. CGPJ. 